\title{
Urban Road Traffic Management - Present and the Future Scenario
}

\author{
Prof. Rajashree Shettar ${ }^{1}$, Prof. Manjunath A. $E^{2}$ \\ ${ }^{I}$ (Department of Computer Science and Engineering, R. V College of Engineering, India) \\ ${ }^{2}$ (Department of Computer Science and Engineering, $R$. V College of Engineering, India)
}

\begin{abstract}
Effective traffic management is the need of the hour to solve issues related to transportation of vehicles on roads considering the environment, technology, government policies, etc. Vehicles either two or four wheeler has been increasing at very phenomenal rate year by year leading to congestion, pollution and wastage of man-hours waiting at signal lights for a longer period leading to loss of productivity, reduced quality of life. This ever growing problem has to be controlled by having an efficient solution. Hence traffic survey and analysis is required to be done to introspect the traffic density and pattern of traffic movement at varying timings of the day and avoiding traffic delays at the junctions. This paper discusses various approaches for handling real time traffic by controlling the traffic signal lights accordingly.
\end{abstract}

Keywords: Traffic Congestion, Traffic density, Traffic Signal Controller

\section{Introduction}

Many cities in the world face serious traffic congestion problems on the road leading to tremendous unproductive time losses and increases the probability of accidents. Environment hazards are also caused due to air pollution. Road intersections or junctions could be considered as potential point for conflict. To reduce such conflicts, lot of effort is required for building proper road infrastructure, providing safety rules and regulations, training the drivers to follow the road safety rules. The traffic congestion at the junctions or intersection area could be minimized by controlling the duration of the traffic light.

The need of the hour for handling traffic congestion has resulted in many techniques and solutions. Traffic signals retiming is one of the solution that has proven to reduce the traffic delay. The most challenging part of the urban traffic controlling is that it has varying traffic density. Traffic signal retiming could be executed by analyzing historical data, adaptive signal time based traffic in real time, traffic cycle time, adjustment of the split and offset time [1]. Total time spent by the vehicles waiting needs to be calculated along with mean delay and average vehicle speed of the vehicles. The current traffic signals at various cities operate in a fixed mode irrespective of the vehicle density. To tackle this problem, variable traffic signal lights has to be designed (adaptive) based on the density of the vehicles using surveillance techniques and inputs from traffic management centers in order to meet changing requirements [1-8]. Data collected with respect to traffic helps to take decisions related to infrastructure and maintenance of transport system. Data variations in traffic could be recorded on an hourly basis, cyclic basis or on a daily basis. Surveys help in estimating of accuracy by collecting relevant data on the number and type of vehicles passing through a specified point on a link or at junctions. The number of people could also help in finding out how many people are using the road [9]. Unit of measurement for volume of traffic is vehicles per hour (vehicles/hour), peak hour demand on road (vehicles/day), annual average daily traffic. In urban areas traffic volume is high during start and end of the day.

Traffic surveys are conducted based on certain critical parameters such as density, speed and direction of flow of the traffic. Video monitoring and surveillance has been extensively used to monitor and estimate traffic density using image processing techniques. To alleviate urban traffic congestion several factors needs to be considered. Traffic volume surveys could be carried out either manually or through automation, a combination of manual and hybrid counts is also done in [9]. Many technologies have been used to detect traffic congestion such as inductive loop, magnetometer, infrared, ultrasonic, sensors, RFID, GSM, etc.

(a) Linear programming

\section{Approaches For Handling Traffic Density}

Van Roy et. al [10] suggest that the count of vehicles could be minimized based on working of traffic light switching schemes. The timing of the traffic lights could be controlled dynamically by counting the total vehicles waiting in the queue. Sampling approach is used to approximate solutions by applying Reduced Linear Program to obtain optimization problems when the constraints are intractable. Probability distribution is considered for solving the problem based on certain constraints. Study of traffic control problem has been executed with constraint sample size, probability measure and traffic arrival rates parameters. It is found that the results are dependent on an ideal value of probability measure for reduced linear programming to work. In 
[11], authors have applied dynamic programming using Markov Decision Process to compute optimal policies for a given model.

\section{(b) Hybrid Neural Networks}

In [1] the authors propose solution to massive urban traffic network for a challenging distributed environment using hybrid neural networks for a multi-agent system. The authors have worked on retiming of traffic signal and this has enabled to reduce traffic delay. Eight different traffic signal plans has been proposed to handle various levels of traffic at the intersection. Results have shown that the use of hybrid Neural Network based multi-agent can cater to large scale increasing traffic network scenario. In [8] authors have developed a multi-agent system using fuzzy neural networks. Neural network is trained online continuously for agent based controller. Results after simulation have shown that as the traffic volume increases, significant improvement in the traffic condition occurs with decrease in the mean delay and mean stoppage. In [12] authors have worked on fuzzy neural networks in combination with genetic algorithms for traffic light control at intersections and bridges. Results show less average waiting time for the vehicles by using this technique.

\section{(c) Genetic Algorithms}

In [13] authors have presented a mechanism to continuously adjust signal timings based on realistic condition of the traffic. Congestion of the traffic is managed by altering the traffic signal adaptively based on traffic rate by using genetic algorithm optimization module. Results show that use of genetic algorithm for real time signal timing is better than the fixed signal timing for total vehicle delay considered. In [14] authors have discussed the use of genetic algorithm to improve the performance of traffic light dynamically at traffic junction. The algorithm is designed to optimize the flow of vehicles through altering the green and red light signal dynamically. Result show that genetic algorithm performs better compared to fixed-time controller. Bruno et al. [15] have proposed the use of multimedia sensor networks through Greedy and Probabilistic Data Collection (PDC) approaches. Simulation results have shown that the Greedy solution consumes less network bandwidth.

\section{(d) Token Based approach}

In [16] authors have suggested solution to handle voluminous traffic congestion at saturated levels and unexpected traffic conditions. The structure of the traffic network and current traffic scenario is considered for prediction by using mixed discrete-continuous planning by controlling traffic light green signals to efficiently reduce congestion of specified roads. Token based approach is used wherein the traffic situation is depicted as a directed graph and intersections is assigned a token. Based on the value of the token, one or more lights, turn green (without any contradiction), with minimum and maximum timing for green light at the intersection.

\section{(e) Vehicular Ad Hoc Networks}

In [17] authors implement real-time intelligent traffic signal controller. Roadside units are used to monitor the density of the traffic. In [17] authors discuss the traffic control through density based signal management using Vehicular Ad Hoc Network (VANETs). Here each node is a vehicle, equipped with wireless communications technology and each node is able to communicate with other nodes or vehicles. Roadside units are used to collect count of the traffic and this is used to change the traffic signal timings dynamically based on low and high density of vehicles. In [18] authors have proposed VANETs at the intersection to collect and consolidate speed of the vehicles on the run and their position. The problem is tackled by applying job scheduling wherein platoon of vehicles are treated as jobs. The paper uses oldest arrival first algorithm and the results have shown that the delay is reduced for vehicles that pass through intersection. The authors use traffic signal controller which has been designed to be adaptive to real-time traffic condition. The information from other vehicles is received by the controller. This information is used to optimize the scheduling of vehicles waiting at the intersection. The main concern in adopting this technology is issues related to connectivity, coverage and energy cost.

\section{Approaches For Improving Traffic Congestion}

To improve the traffic congestion the following approaches could be adopted:

1. To predict the volume of the traffic through data analytics and alerting the road users about the congestion. Information about alternate route and optimum solution to be provided based on data collected via Global Positioning System (GPS), General Packet Radio Service (GPRS), Wireless Local Area Network, etc.

2. Providing online information about the city's current and forecasted traffic situation, with hourly updates by having exclusive web portal.

3. Installations of surveillance cameras and quick response to emergency calls.

4. Need for automation and integration of infrastructure with hardware, software, use of digital telecommunication networks, embedded intelligence, sensors and optimized solutions. 
5. To provide environmental information and safety.

6. Incident detection systems to warn the drivers.

7. Improving the infrastructure by widening of the roads

\section{Future Scenario}

Use of mass public transport such as buses, metro rails, and trains has helped major cities to a greater extent to reduce traffic congestion. Buses enabled with intelligent transport system with security, having effective and strict parking policies, integrated transport policies to address issues related to infrastructure development, transport, policies to reduce the use of personal vehicles and having exclusive lanes for public transport are some of the factors that help reduce traffic congestion [19].

\section{Conclusion}

This paper has focused on the work carried out for urban traffic management using various technologies. The paper surveys the various solutions proposed by authors for handling voluminous traffic at the junctions. The various technologies used to reduce the density of the traffic at junctions by controlling the signal lights has been explored. The outcome of the survey suggest certain solution to curb the traffic menace by adopting the use of intelligent transport system, using of mass transport, having strict policies and by improving the infrastructure by broadening the roads, forming new roads, by constructing metro rails.

\section{References}

[1]. Deepti Srinivasan, Min Chee Choy, and Ruey Long Cheu, "Neural Networks for Real-Time Traffic Signal Control", IEEE Transactions On Intelligent Transportation Systems, Vol. 7, No. 3, September 2006, pp. 261-272.

[2]. S. Chiu and S. Chand, "Self-organizing traffic control via fuzzy logic," in Proc. 32nd IEEE Conf. Decision Control, 1993, pp. 1987-1902.

[3]. G. Nakamiti and F. Gomide, "Fuzzy sets in distributed traffic control," in Proc. 5th IEEE Int. Conf. Fuzzy Syst., 1996, pp. 16171623.

[4]. S. Mikami and Y. Kakazu, "Genetic reinforcement learning for cooperative traffic signal control," in Proc. 1st IEEE Conf. Evol. Comput., vol. 1, 1994, pp. 223-228.

[5]. W. Wei and Y. Zhang, "FL-FN based traffic signal control," in Proc. FUZZ-IEEE, May 12-17, 2002, vol. 1, pp. $296-300$.

[6]. J. C. Spall and D. C. Chin, "Traffic-responsive signal timing for system wide traffic control," Transp. Res.-C, vol. 5, no. 3/4, pp. 153-163, 1997.

[7]. E. Bingham, "Reinforcement learning in neural fuzzy traffic signal control," Eur. J. Oper. Res., vol. 131, no. 2, pp. 232-241, Jun. 2001.

[8]. M. C. Choy, D. Srinivasan, and R. L. Cheu, "Cooperative, hybrid agent architecture for real-time traffic control," IEEE Trans. Syst., Man, Cybern. A, Syst., Humans, vol. 33, no. 5, pp. 597-607, Sep. 2003.

[9]. Urban Road Traffic Surveys, ODA overseas road note, Transport Researc Laboratory- A report pp.1-64.

[10]. Farias, D.P. de, Benjamin van Roy, "On Constraint sampling in the Linear Programming Approach to Approximate Dynamic Programming", Mathematics of operations research, Vol. 29, (3), August 2004, pp. 462-278.

[11]. Haijema, R, Jan van der Wal, "An MDP decomposition approach for traffic control at isolated signalized intersections", Vol. 40, (2), May 2006, pp. 484-496.

[12]. J Venkatesh et. al, Traffic Signal Control Based On Fuzzy Artificial Neural Networks With Particle Swarm Optimization", International Journal of New Technologies in Science and Engineering Vol. 3,(7), July 2016, pp. 20-25.

[13]. Jinwoo Lee, Baher Abdulhai, Amer Shalaby \& Eui-Hwan Chung, "Real-Time Optimization for Adaptive Traffic Signal Control Using Genetic Algorithms", Aug 2006, pp. 111-122.

[14]. Ayad M. Turky, M. S. Ahmad, M. Z. M. Yusoff, Baraa T. Hammad, "Using Genetic Algorithm for Traffic Light Control System with a Pedestrian Crossing", 2009, pp 512- 519.

[15]. Bruno R., Nurchis M. Robust and efficient data collection schemes for vehicular multimedia sensor networks, Proceedings of the IEEE 14th International Symposium and Workshops on World of Wireless, Mobile and Multimedia Networks (WoWMoM), Madrid, Spain. 4-7 June 2013, pp. 1-10.

[16]. Mauro Vallati, Daniele Magazzeni, Bart De Schutter, Lukas Chorpa and Thomas L. McCluskey, "Efficient Macroscopic Urban Traffic Models for Reducing Congestion: A PDDL+ Planning Approach", Proceedings of the Thirtieth AI Conference on Artificial Intelligence (AAAI-16), 2016, pp. 3188-3194.

[17]. Shweta N. Pable, Amit Welekar, "Literature Survey on Density Based Signal Management in Traffic System", International Journal of Computer Applications, 2014, pp. 0975 - 8887.

[18]. Kartik Pandit, Ghosal, D., Zhang, H.M., Chen-Nee Chuah, "Adaptive Traffic Signal Control With Vehicular Ad hoc Networks", IEEE Transactions on Vehicular Technology, Vol. 62, (4), May 2013

[19]. M. Absar Alam and Faisal Ahmed, "Urban Transport Systems and Congestion: A Case Study Of Indian Cities", Transport and Communications Bulletin for Asia and the Pacific, No. 82, 2013, pp. 33-43. 